\title{
SUR LES FEUILLETAGES HOLOMORPHES SINGULIERS DE CODIMENSION 1
}

\author{
BOUCHRA GMIRA
}

\begin{abstract}
Let $\mathcal{F}$ be a codimension one holomorphic foliation which singular set $\Sigma$ is contained in a compact lear $S$ of $\mathcal{F}$.

When $\mathcal{F}$ is of dimension one, $\Sigma$ is a set of isolated points $\left\{q_{1}, \ldots, q_{r}\right\}, \mathrm{C}$. Camacho and P. Sad define the index of $\mathcal{F}$ at each point $q_{k}$ and prove that the sum of these indices equais the Euler class $c_{1}(E)$ of the fibre bundle $E$ normal to $S$.

Generally when ever $\Sigma$ is of any dimension $m$, we cas define a such index $i_{\alpha}$ along the maximal dimension strates $\left\{\Sigma_{\alpha}\right\}$ of a suitable stratification of the complex variety $\Sigma$. Let $\sigma_{\alpha}$ be the fondamental cycle of $\bar{\Sigma}_{\alpha}, \sigma$ the 2 m-cycle of $S$ defined by $\sigma=$ $\sum i_{\alpha} \cdot \sigma_{\alpha}$ and $\sigma^{*}$ the 2-cocycle dual to $\sigma$ by Poincaré isomorphism $\stackrel{\alpha}{H^{2}}(S) \rightarrow H_{2 m}(S)$, we prove that the cohomology class $\left[\sigma^{*}\right]$ equals the Euler class $c_{1}(E)$.
\end{abstract}

\section{Introduction.}

Soit $S$ une variété analytique complexe compacte de dimension (complexe) $m+1$, plongée dans une variété analytique complexe $M$ de dimension $m+2$.

Notons $\mathcal{F}$ un feuilletage holomorphe de codimension 1 de $M$ admettant un ensemble singulier $\Sigma \subset S$ tel que :

(i) $\Sigma$ est un ensemble analytique complexe de codimension 1 dans $S$, (ii) $S-\Sigma$ est une feuille de $\mathcal{F}$.

Dans le cas $m=0, \Sigma$ est un ensemble de points isolés $\left\{q_{1}, \ldots, q_{r}\right\}$. C. Camacho et $\mathrm{P}$. Sad définissent l'indice de $\mathcal{F}$ en chaque point de $\Sigma$ et montrent que la somme de ces indices est égale à la classe d'Euler du fibré normal à $S$ lou plutôt à son évaluation sur le cycle fondamental $[S])[4]$.

Dans le cas général, nous montrons que l'on peut définir un tel indice $i_{\alpha}$ le long des strates $\left\{\Sigma_{\alpha}\right\}$ de dimension maximale $m$ d'une stratification 
convenable de $\Sigma$ en variétés analytiques complexes. Dans une carte locale de $M$, au voisinage d'un point $q$ d'une strate $\Sigma_{\alpha}$, il est possible de se représenter cet indice comme l'indice de C. Camacho et $P$. Sad sur la trace de la configuration $\left(S, \Sigma_{\alpha}, \mathcal{F}\right)$ par un 2-plan complexe transverse à cette configuration. Notons $\sigma_{\alpha}$ le cycle fondamental de la variété (singulière) $\bar{\Sigma}_{\alpha}$ et considérons le $2 m$-cycle de $S: \sigma=\sum_{\alpha} i_{\alpha} \sigma_{\alpha}$. Nous montrons le résultat suivant :

Théorème. Soit $E$ le fibré normal à $S$ dans $M, c_{1}(E) \in H^{2}(S)$ sa classe d'Euler, alors

$$
c_{1}(E)=\left[\sigma^{*}\right]
$$

où $\left[\sigma^{*}\right]$ est la classe du 2-cocycle dual de $\sigma$ par isomorphisme de Poincaré $H^{2}(S) \stackrel{\cong}{\longrightarrow} H_{2 m}(S)$.

Ce résultat figurait déjà dans [5], avec des hypothc̀ses restrictives portant sur la variété $S$. Puis J.P. Brasselet en a donnć une démonstration levant ces hypothèses dans [3] et A.L. Nèto en a donné une autre démonstration comme cas particulier d'un résulat, plus général ([9, Théorème 1]). La présente démonstration est plus géométrique et plus proche des idées de Bott sur les feuilletages singuliers. Je remercie vivement J.P. Brasselet pour les remarques et suggestions qu'il m'a faites tout au long de ce travail.

\section{Définition de I'indice $i_{\alpha}$.}

Considérons une stratification de $\Sigma$ en variétés analytiques complexes, et notons $\Sigma_{\alpha}$ une strate de dimension maximale $m$. Soit $\Phi: \mathcal{U} \rightarrow \mathrm{C}^{m} \times$ $\mathrm{C} \times \mathrm{C}$ une carte locale de $M$ au voisinage d'un point de $\Sigma_{\alpha}$, telle que $\Phi(\mathcal{U} \cap S) \subset\{z=0\}$ et $\Phi\left(\mathcal{U} \cap \Sigma_{\alpha}\right) \subset\{y=z=0\}$. Soit $\eta$ une 1-forme intégrable qui définit $\mathcal{F}$ dans $\mathcal{U}$, alors $\eta$ s'écrit, dans la carte $(\mathcal{U}, \Phi)$, sous la forme :

$$
\eta=z A(x, y, z) d x+z B(x, y, z) d y+(z C(x, y, z)+D(x, y)) d z
$$

où $(x, y, z) \in \mathrm{C}^{m} \times \mathbf{C} \times \mathbf{C}, D(x, y)=a(x) y^{n}+\cdots, n \geq 1$ et $a(0) \neq 0$.

Soit $P$ un 2-plan complexe d'équation $x=$ constante, transverse à $S$ et à $\Sigma_{\alpha}$ dans $\mathcal{U}$, la trace de $\mathcal{F}$ sur $P$ est définie, dans la carte $(\mathcal{U}, \Phi)$, par la forme différentielle :

$$
\eta_{x}=z B(x, y, z) d y+(z C(x, y, z)+D(x, y)) d z
$$

On pose :

$$
i(x)=-\operatorname{Rés}_{y=0}\left(\left.\frac{\partial}{\partial z}\left[\frac{z B(x, y, z)}{z C(x, y, z)+D(x, y)}\right]\right|_{z=0}\right) .
$$


Il est facile de voir que

$$
i(x)=-\underset{y=0}{\operatorname{Rés}} \frac{B(x, y, 0)}{D(x, y)}
$$

et que $i(x)$ dépend analytiquement de $x \in C$, lorsque $a(x) \neq 0$.

Lemme 1. La dérivée $i^{\prime}(x)$ de la fonction $i(x)$ est nulle.

Isa condition d'intégrabilité de $\eta$ donne, pour $z=0$,

$$
\frac{\partial}{\partial y}\left(-\frac{A}{D}\right)+\frac{\partial}{\partial x}\left(\frac{B}{D}\right)=0
$$

En se plaçant sur unc courbe convenable $\gamma$, de façon à pouvoir calculer les résidus, on obtient :

$$
\frac{1}{2 i \pi} \int_{\gamma} \frac{\partial}{\partial y}\left(-\frac{A}{D}\right) d y+\frac{1}{2 i \pi} \int_{\gamma} \frac{\partial}{\partial x}\left(\frac{B}{D}\right) d y=0
$$

d'où :

$$
\int_{\gamma} \frac{\partial}{\partial y}\left(-\frac{A}{D}\right) d y+\frac{\partial}{\partial x} \int_{\gamma}\left(\frac{B}{D}\right) d y=0
$$

et $i^{\prime}(x)=\frac{1}{2 i \pi} \int_{\gamma} \frac{\partial}{\partial y}\left(-\frac{A}{D}\right) d y=0$.

Lemme 2. L'indice $i(x)$ est indépendant du choix de la forme différentielle $n$.

En effet, si $\tilde{\eta}=z \tilde{A} d x+z \tilde{B} d y+(z \tilde{C}+\tilde{D}) d z$ est une autre forme qui définit le feuilletage $\mathcal{F}$ dans $\mathcal{U}$, alors $\hat{\eta}=g \eta$, ò̀ $g$ ne s'annule pas, et done

$$
\frac{\tilde{B}(x, y, 0)}{\tilde{D}(x, y)}=\frac{B(x, y, 0)}{D(x, y)}
$$

d'où le lemme.

Remarque. On vérifie facilement aussi qu'ur changement de coordonnées dans (1) par un diffcomorphisme local $x=f(u, v, 7 u), y=$ $v g(u, v, w), z=w h(u, v, w)$ envoyant $\{w=0\}$ sur $\{z=0\}$ et $\{v=0\}$ sur $\{y=0\}$, ne change pas le résidu. 
Corollaire. Soit $x$ un point de $\Sigma_{\alpha} \cap U$, l'indice $i(x)$ ne dépend que du feulletage $\mathcal{F}$ au voisinage de $\Sigma_{\alpha}$.

Notations. Nous avons vu plus haut que l'indice $i(x)$ est localement constant sur $\Sigma_{\alpha}$, il est donc constant sur chaque strate connexe, en dehors d'un sous-ensemble analytique de mesure nulle $Q_{\alpha}$ (non nécessairement connexe). On sous-stratifie alors $\Sigma$ de façon à ce que les strates de dimension maximum $m$ soient maintenant les $\Sigma_{\alpha}-Q_{\alpha}$, que l'on se permettra encore d'appeler $\Sigma_{\alpha}$ dans la suite. La valeur de l'indice $i(x)$ le long de $\Sigma_{\alpha}$ sera notée $i_{\alpha}$. Si $\sigma_{\alpha}$ désigne le cycle fondamental de la variété (singulière) $\bar{\Sigma}_{\alpha}$, alors $\sigma=\sum_{\alpha} i_{\alpha} \sigma_{\alpha}$ définit un $2 n$-cycle de $S$.

\section{Résidus des connexions à singularités.}

Soit $G$ un groupe de Lie, $\mathcal{G}$ son algèbre de Lite et $\pi: E \rightarrow S$ un $G$ fibré principal différentiable. Pour $k$ fixé, on note $I^{k}(G)$ l'espace vectoriel des polynomes homogènes de degrc $k$ sur $\mathcal{G}$ invariants par la représentation adjointe de $G$ sur $\mathcal{G}$ et $A_{b n}^{k}(S)$ l'espace vectoriel des $k$ formes différentielles sur $S$ à valeurs complexes (Dans la suite, on prendra $G=G L(1, \mathrm{C})$ et on se permettra de noter aussi $\pi: E \rightarrow S$ le $G L(1, \mathrm{C})$ fibré principal associé au fibré normal de $S$ dans $M$ ].

Définition ([6, Vol II, Chap. XII pp. 233-294]). Soit $\nabla$ une connexion sur $E, \omega$ sa forme de connexion et $\Omega$ sa forme de courbure. Si $P \in I^{1}(G)$, on définit une 2-forme à valeurs complcxes sur $E$, cn posant :

$$
P(\Omega)\left(X_{1}, X_{2}\right)=\frac{1}{2}\left[P\left(\Omega\left(X_{1}, X_{2}\right)\right)-P\left(\Omega\left(X_{2}, X_{1}\right)\right)\right]
$$

Soit $\bar{P}(\Omega)$ l'unique 2-forme fermée sur $S$ telle que:

$$
\pi^{*}(\bar{P}(\Omega))=P(\Omega)
$$

en posant $\lambda_{\omega}(P)=\bar{P}(\Omega)$, on définit une application :

$$
\lambda_{\omega}: I^{1}(G) \rightarrow A_{D R}^{2}(S) .
$$

L'application induite en cohomologic par $\lambda_{\omega}$ est indépendante de la connexion choisie. C'est l'homomorphisme caractéristique de ChernWeil, on le note $\lambda: I^{1}(G) \rightarrow H_{D R}^{2}(S)$.

Définition ([1, Paragraphe 5]). Soient $\omega_{0}$ et $\omega_{1}$ deux formes de connexion sur $E$, on définit une application :

$$
\Delta_{\omega_{0}, \omega_{1}}: I^{1}(G) \rightarrow A_{D R}^{1}(S)
$$


de la manière suivante : notons $I=[0,1]$, on définit, sur le fibré $E \times I$, de base $S \times I$, une 1-forme $\tilde{\omega}$ à valeurs dans $\mathcal{G}$, en posant :

$$
\begin{cases}\left.\tilde{\omega}\right|_{E \times\{t\}}=t \omega_{0}+(1-t) \omega_{1} & \text { pour } t \in I \\ \left.\tilde{\omega}\right|_{\{x\} \times I}=0 & \text { pour } x \in E\end{cases}
$$

Pour tout polynôme $P \in I^{1}(G)$, on pose alors :

$$
\Delta_{\omega_{0}, \omega,}(P)=\int_{I} \lambda_{\dot{\omega}}(P)
$$

où l'intégrale $f_{t}: A_{D R}^{2}(S \times I) \rightarrow A_{D R}^{1}(S)$ désigne l'intégratution le long de la fibre I.

Considérons maintcnant une triangulation differentiable $(K)$ de $S$ dont le 1-squelette ne rencontre pas un ensemble fini de points $Q$, et notons $c$ un 2-simplexe de cette triangulation. Soit, $s: V_{c} \rightarrow E$ une scction différentiable de $E$ définie au-dessus d'un voisinage $V_{c}$ de $c$ dans $S$. On désigne par $\omega_{s}$ la connexion plate sur $\left.E\right|_{V_{c}}$ associćc à la trivialisation définie par $s$ (i.e. $s^{*}\left(\omega_{s}\right)=0$ ). Soi $\omega$ unc conncxion définie sur $\left.E\right]_{s-c}$ (connexion à singularités).

Définition $([7])$. A tout simplexe $c$ de $(K)$, et ì tout polynôme $P \in$ $I^{1}(G)$ on associc lc nombre

$$
\operatorname{Rés}_{e}(\omega, P)=\int_{\partial c} \Delta_{\omega_{y}, \omega}(P)
$$

appelé résidu sur $c$ de $\omega$, relativement à $P$. On définit airisi unc applicàtion linéaire

$$
\operatorname{Rés}(\omega): I^{1}(G) \rightarrow C^{2}(K, \mathrm{C})
$$

en posant $\langle\operatorname{Rós}(\omega) P, c\rangle=\operatorname{Rés}_{c}(\omega, P)$ (ici, $C^{2}(K, \mathrm{C})$ désigne l'onscrnble des 2-cochaînes simpliciales sur $(K)$, à coefficients complexes).

Si l'on note maintenant $I: A_{O R}^{*}(S) \rightarrow C^{*}(K, \mathrm{C})$ l'intégration des formes différenticlles, on a :

$$
<J(\alpha), c>=\int_{c} \alpha
$$

Rappelons que $J$ induit, en cohomologie, un isomorphisme, d'algèbres graduées

$$
[J]: H_{D R}^{*}(S) \stackrel{\cong}{\longrightarrow} H^{*}(K, \mathrm{C})
$$


Théorème des résidus. ([7])

(i) L'application Rés $(\omega)$ prend ses valeurs dans l'espace $Z^{2}(K, \mathrm{C})$ des cocycles de $C^{2}(K, \mathbf{C})$,

(ii) Notant $[R e ́ s(\omega)]: I^{1}(G) \rightarrow H^{2}(K, \mathrm{C})$ l'application induite en cohomologie, la composée $[J]^{-1} \circ[R e ́ s(\omega)]$ coinncide avec l'homomorphisme caractéristique de Chern-Weil

$$
\lambda: I^{1}(G) \rightarrow H_{D R}^{2}(S) .
$$

\section{Indice et rêsidu de connexions à singularités.}

On sait que tout 2-cycle de $S$ est réalisable par une sous-variété différentiable $W$ de $S$ ([10, Théorème II.27]), que l'on peut supposer transverse à $\Sigma$ (Thcócòme de transversalité de Thom [8]). La variété $W$ ne rencontre donc que les strates de dimcrision maximale de $\Sigma$, et ceci en des points isolés $Q=\left\{q_{1}, \ldots, q_{r}\right\}=\Sigma \cap W$.

Au voisinage de chaque poirt $q_{k}$ de $\Sigma \cap W$, on pcut rnodifier la variété $W$ de façon à obtenir unc variété différentiable $W^{\prime}$ représcritant un cycle homologue à $W$ et telle que chaque point $q_{k}$ admette, dans $W^{\prime}$, un voisinage $U_{k}$, disque analytique. Pour cela, on considère, pour tout point $q_{k}$, un tel disque $U_{k}$, déformation d'un voisinage $V_{k}$ de $q_{k}$ dans $W$. La déformation (supposéc différentiable) fournit l'homologie cherchéc. De plus, on peut supposer que les disques $U_{k}$ sont disjoints et que chacun d'eux est contenu dans un ouvert trivialisant du fibré normal à $S$ dans $M$.

Par abus de notation, la variété $W^{\prime}$ scra encore notée $W$ par la suite.

Soit $T$ un voisinage tubulaire de $S$ dans $M$, identifié à un voisinage de la section nulle du fibré normal de $S$ dans $M$. Le feuilletage $\mathcal{F}$ induit un feuilletage de $T$, cncore noté $\mathcal{F}$. En restriction à $L=S-\Sigma$, le fibré normal à $\mathcal{F}$, appelé $\nu$, s'identifie au fibré normal de $S$ dans $M$; soit $p:\left.\nu\right|_{L} \rightarrow L$ sa projection. Enffin, on notera $\pi: E \rightarrow S$ la projection du $G L(1, \mathrm{C})$-fibré principal associé a fibré normal de $S$ dans $M$.

Dans un système de coordonrées locales appropriées, $p^{-1}\left(U_{k}\right)$ s'identifie à un 2-plan complexe de $M$. La trace de $\mathcal{F}$ sur $p^{-1}\left(U_{k}\right)$ est alors définie par une 1-forme holomorphe $\eta_{k}$ telle que (2), nous la noterons :

$$
\eta_{k}(y, z)=B_{k}(y, z) d y+C_{k}(y, z) d z
$$

avec $(y, z) \in U_{k} \times \mathbf{C}^{*}, B_{k}(0,0)=C_{k}(0,0)=0$ et $B_{k}(y, 0)=0$.

Soit $\theta_{k}$ la 1-forme défnie sur $U_{k}-\left\{q_{k}\right\}$ par

$$
\theta_{k}(y)=\frac{\frac{\partial B_{k}}{\partial z}(y, 0)}{C_{k}(y, 0)} d y
$$


alors $i\left(q_{k}\right)=-$ Rés $\theta_{k}=-\frac{1}{2 i \pi} \int_{\partial \bar{U}_{k}} \theta_{k}$.

Lemme 3. Il existe une connexion $\nabla$ sur $\left.E\right|_{S-\Sigma}$ telle que sa restriction $\grave{a} U_{k}-\left\{q_{k}\right\}$ soit définie par la 1-forme $\theta_{k}$. C'est la restriction $\grave{a}$ $S-\Sigma$ de la connexion de Bott (qui est parfaitement défnie sur $M-\Sigma$ en tant que connexion partielle).

Démonstration: Soit comme ci-dessus, $T$ un voisinage tubulaire de $S$ dans $M$. Notons encore $\mathcal{F}$ le feuilletage de $M$ restreint à $T, T T$ le fibré tangent à $\mathcal{T}, \tau$ le fibré tangent au feuilletage $\mathcal{F}$ et $\nu$ le fibré normal à $\mathcal{F}$. Nous avons une suite exacte de fibrés au dessus de $\mathcal{T}-\Sigma$ :

$$
\left.\left.\left.0 \longrightarrow \tau\right|_{T-\Sigma} \longrightarrow T \mathcal{T}\right|_{T-\Sigma} \stackrel{h}{\longrightarrow} \nu\right|_{T-\Sigma} \longrightarrow 0
$$

qui induit, au dessus de $L=S-\Sigma$, la suite exacte de fibrés :

$$
\left.\left.\left.0 \longrightarrow \tau\right|_{L} \longrightarrow T T\right|_{L} \stackrel{k}{\longrightarrow} \nu\right|_{L} \longrightarrow 0
$$

Le fibré $\left.\tau\right|_{L}$ n'est autre que le fibré tangent à la feuille $L$, on le note $T L$, $\left.\nu\right|_{L}$ est le fibré normal à $L$ et associé au $G L(1, C)$-fibré principal $\left.E\right|_{L}$. Si $\mathcal{O}(S)$ désigne l'algèbre des fonctions holomorphes sur $S, \Gamma(T L)$ et $\Gamma\left(\left.\nu\right|_{L}\right)$ désignant respectivement les $\mathcal{O}(S)$-modules des sections de $T L$ et $\left.\nu\right|_{L}$, nous définissons une connexion $\nabla$ sur $\left.E\right|_{L}$ en posant :

$$
\nabla_{X}(Z)=h[X, Z] \quad \forall X \in \Gamma(T L) \text { et } \forall Z \in \Gamma\left(\left.\nu\right|_{L}\right)
$$

([1, Paragraphe 6]).

Regardons comment se spécialise cette connexion $\nabla$ lorsqu'on se restreint à l'un des disques épointés $U_{k}-\left\{q_{k}\right\}$. Notons $\mathcal{T}_{k}$ la restriction de $\mathcal{T} \grave{a} U_{k}-\left\{q_{k}\right\}, \mathcal{F}_{k}$ la trace du feuilletage $\mathcal{F}$ sur $\mathcal{T}_{k}, T \mathcal{T}_{k}$ le fibré tangent à $\mathcal{T}_{k}, \tau_{k}$ le fibré tangent à $\mathcal{F}_{k}$ et $\nu_{k}$ le fibré $\nu$ restreint à $\mathcal{T}_{k}$. Nous avons une suite exacte de fibrés restreints à $U_{k}-\left\{q_{k}\right\}$ :

$$
0 \longrightarrow \tau_{k} \longrightarrow T \mathcal{T}_{k} \stackrel{h}{\longrightarrow} \nu_{k} \longrightarrow 0
$$

Une base de $\Gamma\left(\tau_{k}\right)$ est donnée par

$$
X_{k}=-C_{k}(y, 0) \frac{\partial}{\partial y}
$$

et le champ de vecteurs $\frac{\partial}{\partial x}$ constitue une base de $\Gamma\left(\left.\nu\right|_{U_{k}-\left\{q_{k}\right\}}\right)$. 
Le champ

$$
Y_{k}=-C_{k}(y, z) \frac{\partial}{\partial y}+B_{k}(y, z) \frac{\partial}{\partial z} \quad(y, z) \in\left(U_{k}-\left\{q_{k}\right\}\right) \times \mathbf{C}
$$

est défini dans un voisinage de $U_{k}-\left\{q_{k}\right\}$ dans $T$ et sa restriction à $L$ est $X_{k}$.

Soit $\omega$ la 1-forme de connexion qui définit $\nabla$, elle vérifie la relation

$$
\nabla_{X_{k}}\left(\frac{\partial}{\partial z}\right)=\omega\left(X_{k}\right) \frac{\partial}{\partial z}
$$

or

$$
\begin{aligned}
\nabla_{Y_{k}}\left(\frac{\partial}{\partial z}\right) & =h\left(\left[-C_{k}(y, z) \frac{\partial}{\partial y}+B_{k}(y, z) \frac{\partial}{\partial z}, \frac{\partial}{\partial z}\right]\right) \\
& =h\left(\frac{\partial C_{k}}{\partial z}(y, z) \frac{\partial}{\partial y}-\frac{\partial B_{k}}{\partial z}(y, z) \frac{\partial}{\partial z}\right)=-\frac{\partial B_{k}}{\partial z}(y, z) \frac{\partial}{\partial z}
\end{aligned}
$$

et

$$
\nabla_{X_{k}}\left(\frac{\partial}{\partial z}\right)=-\frac{\partial B_{k}}{\partial z}(y, 0) \frac{\partial}{\partial z}
$$

En comparant (3) et. (4), il vicrit, par abus de notation

$$
\omega=\frac{\frac{\partial B_{k}}{\partial z}(y, 0)}{C_{k}(y, 0)} d y=\theta_{k} \quad \text { sur } U_{k}-\left\{q_{k}\right\}
$$

d'où le lemme.

Nous pouvons supposer que $W$ est un sous-complexe de la triangulation $(K)$ de $S$. On note $\left(K_{W}\right)$ la triangulation induite sur la variété $W$. Son 1-scuuclette ne rencontre pas l'ensemble $\Sigma \cap W=\left\{q_{1}, \ldots, q_{r}\right\}$. On peut aussi supposer que chaque point $q_{k}$ est situé dans un 2-simplexe $c_{k}$ de $\left(K_{W}\right)$, lui-mêrne situé dans le disque $U_{k}$.

Lemme 4. On a

$$
i\left(\eta_{k}\right)=\operatorname{Rés}_{c_{k}}\left(\omega, P_{1}\right)
$$

où $P_{1} \in I^{1}(G L(1, \mathrm{C}))$ est le polynôme caractéristique associé à la classe d'Euler c $(E)$.

En fait, $P_{3}(u)=\frac{-1}{2 i \pi} u,([6$, Chap. XII section 3] $)$. 
Démonstration: Soit $q_{k}$ un point de $\Sigma \cap W$ et $c_{k}$ le 2-simplexe de la triangulation $\left(K_{W}\right)$ contcrant ce point $\left(c_{k} \subset U_{k}\right)$. On note $s_{k}: V_{c_{k}} \rightarrow E$ une section différentiable de $E$ définie au dessus d'un voisinage $V_{c_{k}}$ du simplexe $c_{k}$ dans $U_{k}$. Désignons par $\omega_{k}$ la conncxion plate sur $E \mid v_{c_{k}}$ définie par $s_{k}$ (elle est caractérisóc par $s_{k}^{*}\left(\omega_{k}\right)=0$, do plus $d \omega_{k}=0$ puisque la connexion est plate). Notons $\omega$ la 1 -forme de connexion de la connexion définie au lernme 3 . Soit $\tilde{\omega}$ la 1 -forme associée aux 1-formes de connexion $\omega_{k}$ ct $\omega$ comme ci-dessus $(\S 3)$ et $\tilde{\Omega}$ la 2 -forme de courbure défnie par $\tilde{\omega}$ (noter que $\tilde{\omega}$ est définic sur $\left.\left.(E \times I)\right|_{\left(V_{c_{k}}-\left\{q_{k}\right\}\right) \times I}\right)$. Nous avons

$$
\bar{\Omega}=d \bar{\omega}=d\left(t \omega_{k}+(1-t) \omega\right)=(1-t) d \omega+\omega \wedge d t-\omega_{k} \wedge d t .
$$

Si $p: E \times I \rightarrow S \times I$ désignc la projection du fibré $E \times I$ sur sa base, alors $r_{k}=\left(s_{k}\right.$, id $\left.l\right): V_{c_{k}} \times I \rightarrow E \times I$ est unc scction du fibré $\left.(E \times I)\right|_{v_{c_{k}} \times I}$ ct

$$
\lambda_{\bar{\omega}}\left(P_{1}\right)=r_{\bar{k}}^{*} P_{1}(\tilde{\Omega}),
$$

d'où

$$
\begin{aligned}
\Delta_{\omega_{k}, \omega}\left(P_{1}\right) & =\int_{I} r_{k}^{*} P_{1}(\tilde{\Omega}) \\
& =-\frac{1}{2 i \pi} f_{I} r_{k}^{*}(\tilde{\Omega}) \\
& =-\frac{1}{2 i \pi} f_{I}(1-t) s_{k}^{*}(d \omega)+s_{k}^{*}(\omega) \wedge d t-s_{k}^{*}\left(\omega_{k}\right) \wedge d t
\end{aligned}
$$

Or $s_{k}^{*}\left(\omega_{k}\right)=0, f_{i}(1-t) s_{k}^{*}(d \omega)=0$ (par définition de l'intégration le long de la fibrc) et $s_{k}^{*}(\omega)=\theta_{k}$ (d'après le lemme 3 ), on a donc:

$$
\Delta_{\omega_{k}, \omega}\left(P_{1}\right)=-\frac{1}{2 i \pi} \int_{0}^{1} \theta_{k} \wedge d t=-\frac{1}{2 i \pi} \theta_{k}
$$

et

$$
\operatorname{Rés}_{c_{k}}\left(\omega, P_{1}\right)=-\frac{1}{2 i \pi} \int_{\partial c_{k}} \theta_{k}=i\left(q_{k}\right)
$$

\section{Démonstration du théorème.}

On se propose de montrer que les cocycles $c_{1}(E)$ et $\left[\sigma^{*}\right]$ prennent la mème valeur sur tout 2-cycle $\mu$ de $S$, ce qui démontrera le théorène.

Comme nous l'avons vu plus haut nous poivons représenter tout 2 cycle de $S$ par une sous-variété différentiable $W$ de $S$, transvorse ì 
$\Sigma$ et admettant, en tout point $q k$ de $\Sigma \cap W$, un voisinage analytique $U_{k}$. Comme précédemment (lemme 4 ), on considère une triangulation différentiablc $K W$ de $W$, que l'on peut supposer finie, $W$ étant compacte. Notons $c_{1}, \ldots, c_{n}$ les 2-simplexes de $K_{W}$, convcnablement orientés, les $r$-premiers $c_{1}, \ldots c_{r}$, étant ceux qui contiennent les points $\left\{q_{1}, \ldots, q_{r}\right\}$ de $\Sigma \cap W$ dans leur intérieur. La classe fondamentale $[W]$ de $W$ est celle du cycle $\mu=\sum_{j=1}^{n} c_{j}$ dans $H_{2}(S)$. D'après le lemme 4 , on a

$$
\sum_{k=1}^{r} i\left(q_{k}\right)=\sum_{k=1}^{r} \operatorname{Rés}_{c_{k}}\left(\omega, P_{1}\right)=\sum_{j=1}^{n} \operatorname{Rés}_{c_{j}}\left(\omega, P_{1}\right),
$$

puisque, si $c_{j}$ ne contient pas de point $q_{k}$, alors Rés ${ }_{c_{j}}\left(\omega, P_{1}\right)=0$. Mais

$$
\begin{aligned}
\sum_{j=1}^{n} \operatorname{Rés}_{c_{j}}\left(\omega, P_{1}\right) & =<\operatorname{Rés}(\omega)\left(P_{1}\right), \sum_{j=1}^{\pi} c_{j}> \\
& =<[\operatorname{Rés}(\omega)]\left(P_{1}\right),[W]> \\
& =<[J]^{-1} \circ[\operatorname{Rés}(\omega)]\left(P_{1}\right),[W]> \\
& =<\lambda\left(P_{1}\right),[W]>
\end{aligned}
$$

on a donc, par le lernme 4 ,

$$
\sum_{k=1}^{r} i\left(q_{k}\right)=<\lambda\left(P_{1}\right),[W]>=<c_{1}(E), \mu>.
$$

Calculons maintenant l'évaluation, sur $\mu$, du 2-cocycle $\sigma^{*}$, dual par isomorphisme de Poincaré du $2 m$-cycle $\sigma=\sum_{\alpha} i_{\alpha} \sigma_{\alpha}$.

Pour cela, considérons une triangulation (finie) $(T)$ de $S$, compatible avec la stratification de $\Sigma$ (i.e. tout simplexe ouvert de $(T)$ est situé dans une seule strate). On se donne une décomposition barycentrique $\left(T^{\prime}\right)$ de (T) telle que :

(i) les points $q_{1}, \ldots q_{k}$ sont barycentres de $2 m$-simplexes de $(T)$,

(ii) la subdivision barycentrique $\left(T^{\prime}\right)$ triangule $W$ (ce qui est possible car $W$ est transverse à $\Sigma$ ).

On notera $t_{j}$ les $2 m$-simplexes de $(T)$. Soit. $(D)$ la décomposition cellulaire de $S$ duale de $(T)$, construite à l'aide de la décomposition barycentrique $\left(T^{\prime}\right)$. La 2-cellule duale du simplexe $t_{j}$ sera notée $d_{j}$ et $d_{j}^{*}$ désignera la $(D)$-cochaine duale de la $(D)$-chaine élémentaire $d_{j}$, c'est-àdire telle que

$$
\left.<d_{j}^{*}, d_{i}\right\rangle=\delta_{i, j}
$$


(symbole de Kronecker). D'après les hypothc̀ses, $(D)$ définit une décomposition cellulaire sur $W$.

Remarquons que lorsque $t_{j}$ contient un point $q_{k}$, alors $t_{j}$ est situé dans $\Sigma$, son irtérieur est entièrement contenu dans une strate régulière $\Sigma_{\alpha}$, et dans ce cas la cellule duale $d_{j}$ est sitúc dans $W$, par raison de transversalité. D'autre part, notant $t_{j_{\alpha}}$ les $2 \pi n$-simplexes de $(T)$ conterns dans la strate $\Sigma_{\alpha}$ et munis de leur orientation caronique (celle de $\Sigma_{\alpha z}$ ), le cycle fondamental de la variété singulic̀rc compactc $\bar{\Sigma}_{\alpha}$ s'écrit $\sigma_{\alpha}=$ $\sum_{j_{\alpha}} t_{j_{\alpha}}$. Nous obtenons donc, puisque $\sigma=\sum_{\alpha} i_{\alpha} \sigma_{\alpha}$

$$
\sigma^{*}=\sum_{\alpha} i_{\alpha}\left(\sum_{j_{\alpha}} d_{j_{\alpha}}^{*}\right)
$$

où la somme $\sum_{j_{\alpha}} d_{j_{\alpha}}^{*}$ portc sur les indices $j_{\alpha}$ tels que la 2-cellule $d_{j_{\alpha}}$ est dualc d'un $2 m$-simplexe $t_{j_{\alpha}}$ dont le support $t_{j_{\alpha}}$ est. contenu dans $\Sigma_{\alpha}$ (voir [2]). De plus, comme

$$
\left\langle d_{j}^{*},[W]>= \begin{cases}1 & \text { si } d_{j} \subset W \\ 0 & \text { si } d_{j} \not \subset W\end{cases}\right.
$$

seules les cellules $d_{j_{\alpha}}$ contenant un point $q_{k}$ (commo centro) interviennent. dans l'évaluation $\left\langle\left[\sigma^{*}\right], \mu\right\rangle$ et il vient

$$
<\left[\sigma^{*}\right], \mu>=\sum_{k=1}^{r} i\left(q_{k}\right)
$$

puisque $i\left(q_{k}\right)=i_{\alpha}$ chaque fois que lc point $q_{k}$ est dans $\Sigma_{\alpha}$. On cr déduit, le théorème.

\section{Bibliographie}

1. R. Borr, "Lectures on Characteristic Classes and Foliations," Lecture Notes in Mathematics 27, Springer-Verlag, Berlin, Heidelberg; New-York, 1972.

2. J.P. BinsSliLeT, Définition combinatoire des homomorphismes de Poincaré, Alexander et Thom, pour une pscudovariété, Astéristue 82-83 (1981). 
3. J.P. Brasselet, Conférences à l'IMPA, Rio de Janeiro, Brćsil, non publié (cité dans [9]), 1984.

4. C. CAMACIO AND P. SAD, Invariant varicties through singularitics of holomorphic vector fields, Ann. Math. 115 (1982), 579-595.

5. B. Gmira, Une généralisation d'un théorème de $C$. Camacho et $P$. Sad relatif aux feuilletages holomorphes singuliers, Thèse de IIIòme cycle, Lille, 1984.

6. S. KonaYAsI1 and K. NOMIZU, "Foundations of Differential Geometry," Vol. II, Intersciences, 1969 .

7. D. Leumann, Résidus des connexions à singularités e classes caractéristiques, Ann. Inst. Fourier Grenoble 31 (1981), 83-98.

8. J. MunOn, "Differentiable Topology," Lecture notes, Princeton, 1958.

9. A.L. Niro, Complex codimension one foliations leaving a c submanifold invariant. Dyramical System and bifurcation theory, Rio de Janciro, Pitman Res. Notes Math. Ser. 160, Longman Sci. Tech. Harlow, 1987 (1985), 295-317.

10. R. Tırom, Quelques propriétés globales des variétés différentiables, Commentarii Moth. Helv. 28 (1954), $17 \cdots 86$.

\author{
Untiversité Abdelmálek lissaâdi \\ lacultê des Sciences de Tétolian \\ Départenent de Ma1. hérrlatiquess \\ B.I'. 2121 'Jetowan \\ MAROC
}

Primera versió rebuda el 19 de Mare de 1991, darterc vetsió rebuda el 9 de Maíg de 1991 